\title{
BRCA1 as tumor suppressor: lord without its RING?
}

Andrew EH Elia ${ }^{1,2,3}$ and Stephen J Elledge $e^{* 1,2}$

\begin{abstract}
BRCA1 is a tumor suppressor with critical roles in the maintenance of genomic stability. It encodes a large protein with an amino-terminal RING domain that possesses ubiquitin-ligase activity. Given the occurrence of numerous cancer-causing mutations within its RING domain, investigators have long suspected that BRCA1's ubiquitin ligase is important for its tumor suppression and DNA repair activities. Using genetically engineered mouse models, two recent studies shed light on this age-old hypothesis.
\end{abstract}

Germline mutations in the BRCA1 and BRCA2 genes account for $5 \%$ of all breast cancers and nearly $80 \%$ of families with hereditary breast cancer and ovarian cancer. These genes preserve genomic integrity by playing important roles in multiple DNA damage response pathways, including homologous recombination repair [1]. $B R C A 1$ encodes a large 1,863-amino-acid protein that harbors an amino-terminal RING domain and two tandem carboxy-terminal BRCT domains (Figure 1a). The RING domain functions as an E3 ubiquitin ligase by recruiting an E2 ubiquitin-conjugating enzyme, while the BRCT domains are phosphopeptide recognition modules [2,3] that enable BRCA1 binding to phosphorylated partners such as Abraxas, BACH1, and CtIP [1]. Cancer-causing mutations in patients occur in both the RING and BRCT domains.

BRCA1 is unlike most E3 ubiquitin ligases in that its activity is enhanced by dimerization with the RING domain of a second protein, BARD1. The RING domains of BRCA1 and BARD1 form a four-helix bundle. Of note, the E2 enzyme makes contacts with the RING domain of BRCA1 but not with that of BARD1 (Figure 1b) [4]. The mechanism by which BARD1 promotes BRCA1 ligase activity is therefore unclear but may involve stabilizing a

\footnotetext{
*Correspondence: selledge@genetics.med.harvard.edu

'Department of Genetics, Harvard Medical School, 77 Ave. Louis Pasteur, NRB158, Boston, MA 02115, USA

Full list of author information is available at the end of the article
}

conformation of BRCA1 optimal for E2 binding. Using elegant mouse models, two recent studies have examined the effect of missense RING mutations on the tumor suppression and DNA repair activities of BRCA1 $[5,6]$.

Shakya and colleagues generated mice expressing BRCA1 with the mutation I26A [5]. This mutation abrogates E2 binding (and thus ubiquitin-ligase activity) but allows assembly of the BRCA1/BARD1 heterodimer. Notably, the BRCA1 RING can directly bind at least eight E2 enzymes, all of which support mono-ubiquitination or poly-ubiquitination in vitro, and I26A mutation ablates interaction with each of these E2 enzymes [7]. Surprisingly, the authors found that the I26A mutation in mice prevented tumor formation to the same degree as wildtype BRCA1 in three conditional genetic models. Furthermore, the DNA damage response remained intact with no changes in chromosome stability or sensitivity to genotoxic stress in mouse embryonic fibroblasts. In prior work, the authors had shown this mutation also conferred no changes in homologous recombination or Rad51 foci formation after ionizing radiation in embryonic stem cells [8].

After demonstrating dispensability of E3 ligase activity for tumor suppression, Shakya and colleagues shifted their attention to the BRCT domains. They engineered mice with the mutation S1598F, which is known to disrupt BRCT phosphopeptide binding at the analogous site (S1655) in human BRCA1 [9] and to cause cancer in patients. In contrast to I26, mutation of S1598 resulted in impaired homologous recombination, reduced Rad51 foci formation after ionizing radiation, increased chromosome instability, and hypersensitivity to genotoxic stress in mouse embryonic fibroblasts. Consistent with the elevated genomic instability, the mice developed tumors at an accelerated rate in the same three genetic backgrounds.

In a second study, Drost and colleagues also evaluated the BRCA1 RING domain by generating mice with the distinct mutation C61G, which occurs in breast cancer patients [6]. This mutation disrupts zinc ion binding necessary for stability of the RING structure. Consequently, it abolishes interaction not only with E2 conjugating enzymes but also with BARD1 [4]. In the absence of p53, mice bearing the C61G mutation developed breast tumors at the same rate as BRCA1 null mice. 


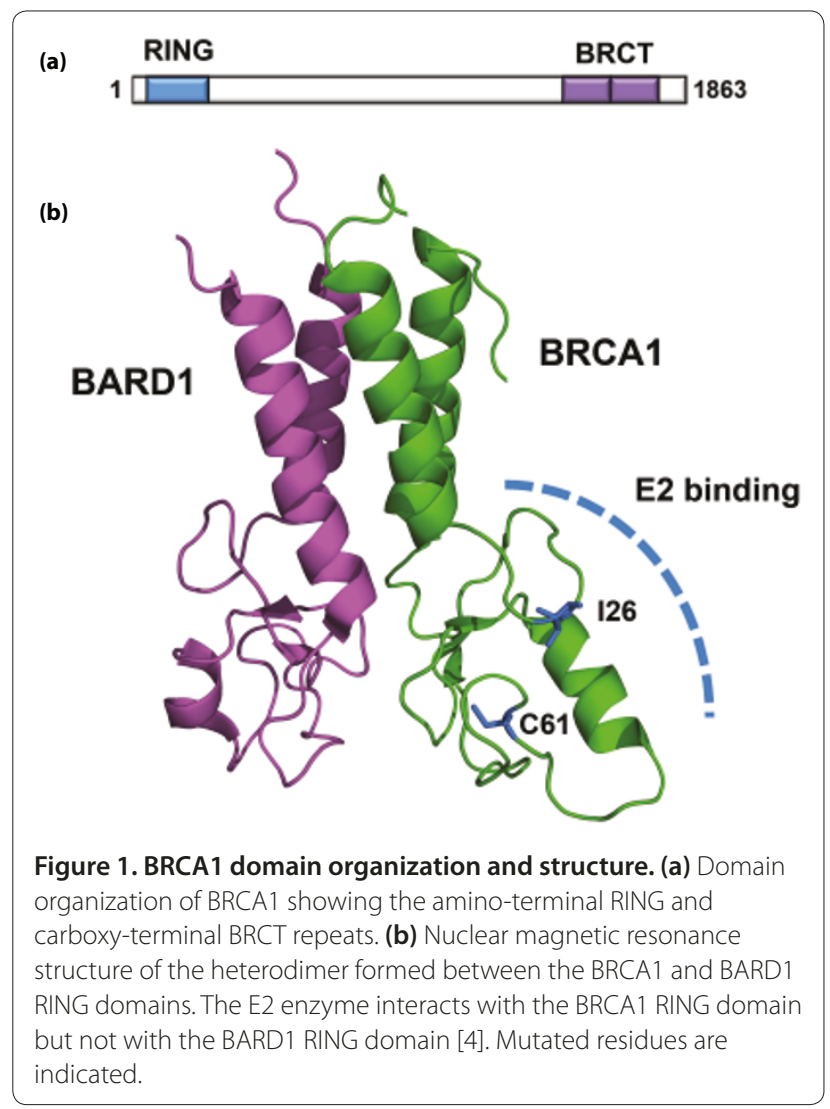

Differences with null mice emerged, however, when examining responses to genotoxic stress, as C61G tumors were less sensitive to both cisplatin and the poly(ADPribose) polymerase inhibitor olaparib. C61G tumors also acquired resistance to cisplatin, whereas $B R C A 1$ null tumors remained responsive over a 250-day period. Because cells from C61G tumors developed more ionizing-radiation-induced Rad51 foci than cells from $B R C A 1$ null tumors, Drost and colleagues proposed that hypomorphic DNA repair activity may account for the acquired resistance. Perhaps hypomorphic repair allows tumors to sustain continued damage until upregulation of a suppressor pathway occurs. To what extent the C61G mutant truly retains hypomorphic activity must be clarified, however, as homologous recombination in this mutant was identical to that in BRCA1 null cells [6].

These two enlightening papers provide new insight into function of the BRCA1 RING domain. Contrary to prior hypotheses, the first study suggests the ubiquitin ligase activity of the RING domain is not essential for BRCA1's roles in genomic maintenance and tumor suppression [5]. Conversely, structural integrity of the RING domain is necessary [6], and this may be related to mediation of tumor suppressor activity by BARD1, independent of BARD1's influence on BRCA1 ligase function. This model is consistent with the finding that BARD1 null mice develop breast cancer [1]. Interestingly, Shakya and colleagues showed that ionizing-radiation-induced formation of conjugated ubiquitin foci (detected by the antibody FK2) was not affected by I26A mutation [5]. However, prior studies have demonstrated that these foci are abrogated by BRCA1 depletion using RNAi [10-12]. Is it possible that BRCA1-dependent ubiquitination at DNA damage foci may be due to a downstream unidentified E3 ligase? Perhaps, but we also cannot rule out the possibility that I26A mutation allows limited in vivo interaction with one of 38 E2 enzymes [13] not previously detected in prior, albeit very rigorous, studies of BRCA1 binding $[7,14]$ and that such interaction is sufficient for ubiquitination of some BRCA1 substrates. Examining whether I26A mutation affects DNA damage-induced ubiquitination of CtIP or other BRCA1 proposed substrates [1] would be informative.

A third study recently suggested that BRCA1 ubiquitinates histone H2A to repress satellite DNA [15]. This study demonstrated that I26A mutation leads to transcriptional derepression of satellite DNA and that addition of exogenous satellite RNA to cells inhibits homologous recombination. Reconciling these results with the normal homologous recombination observed in I26A mutants by Reid and colleagues [8] is difficult. Despite such discrepancies, these new studies reveal intriguing results about BRCA1 biology and set the stage for future work on this complex protein.

\section{Acknowledgements}

AEHE is supported by fellowships from The Jane Coffin Childs Foundation and The American Society for Radiation Oncology. SJE is an investigator with the Howard Hughes Medical Institute.

\section{Competing interests}

The authors declare that they have no competing interests.

\section{Author details}

'Department of Genetics, Harvard Medical School, 77 Ave. Louis Pasteur, Boston, MA 02115, USA. 'Division of Genetics, Brigham and Women's Hospital, 77 Ave. Louis Pasteur, Boston, MA 02115, USA. ${ }^{3}$ Department of Radiation Oncology, Massachusetts General Hospital, 100 Blossom St., Boston, MA 02114, USA.

Published: 10 April 2012

\section{References}

1. Huen MS, Sy SM, Chen J: BRCA1 and its toolbox for the maintenance of genome integrity. Nat Rev Mol Cell Biol 2010, 11:138-148.

2. Manke IA, Lowery DM, Nguyen A, Yaffe MB: BRCT repeats as phosphopeptide-binding modules involved in protein targeting. Science 2003, 302:636-639.

3. Yu X, Chini CC, He M, Mer G, Chen J: The BRCT domain is a phospho-protein binding domain. Science 2003, 302:639-642.

4. Brzovic PS, Keeffe JR, Nishikawa H, Miyamoto K, Fox D 3rd, Fukuda M, Ohta T, Klevit R: Binding and recognition in the assembly of an active BRCA1/ BARD1 ubiquitin-ligase complex. Proc Natl Acad Sci U S A 2003, 100:5646-5651.

5. Shakya R, Reid $\sqcup$, Reczek CR, Cole F, Egli D, Lin CS, deRooij DG, Hirsch S, Ravi K, Hicks JB, Szabolcs M, Jasin M, Baer R, Ludwig T: BRCA1 tumor suppression depends on BRCT phosphoprotein binding, but not its E3 ligase activity. Science 2011, 334:525-528. 
6. Drost R, Bouwman P, Rottenberg S, Boon U, Schut E, Klarenbeek S, Klijn C, van der Heijden I, van der Gulden H, Wientjens E, Pieterse M, Catteau A, Green P, Solomon E, Morris JR, Jonkers J: BRCA1 RING function is essential for tumor suppression but dispensable for therapy resistance. Cancer Cell 2011, 20:797-809

7. Christensen DE, Brzovic PS, Klevit RE: E2-BRCA1 RING interactions dictate synthesis of mono- or specific polyubiquitin chain linkages. Nat Struct Mol Biol 2007, 14:941-948.

8. Reid L, Shakya R, Modi AP, Lokshin M, Cheng JT, Jasin M, Baer R, Ludwig T: E3 ligase activity of BRCA1 is not essential for mammalian cell viability or homology-directed repair of double-strand DNA breaks. Proc Natl Acad SCi USA 2008, 105:20876-20881.

9. Clapperton JA, Manke IA, Lowery DM, Ho T, Haire LF, Yaffe MB, Smerdon SJ: Structure and mechanism of BRCA1 BRCT domain recognition of phosphorylated BACH1 with implications for cancer. Nat Struct Mol Biol 2004, 11:512-518.

10. Morris JR, Solomon E: BRCA1:BARD1 induces the formation of conjugated ubiquitin structures, dependent on $\mathrm{K} 6$ of ubiquitin, in cells during DNA replication and repair. Hum Mol Genet 2004, 13:807-817.

11. Polanowska J, Martin JS, Garcia-Muse T, Petalcorin MI, Boulton SJ: A conserved pathway to activate BRCA1-dependent ubiquitylation at DNA damage sites. EMBO J 2006, 25:2178-2188.
12. Zhao GY, Sonoda E, Barber $L$, Oka H, Murakawa Y, Yamada K, Ikura T, Wang X, Kobayashi M, Yamamoto K, Boulton SJ, Takeda S: A critical role for the ubiquitin-conjugating enzyme Ubc13 in initiating homologous recombination. Mol Cell 2007, 25:663-675.

13. Ye Y, Rape M: Building ubiquitin chains: E2 enzymes at work. Nat Rev Mol Cell Biol 2009, 10:755-764.

14. Christensen DE, Klevit RE: Dynamic interactions of proteins in complex networks: identifying the complete set of interacting E2s for functional investigation of E3-dependent protein ubiquitination. FEBS J 2009, 276:5381-5389.

15. Zhu Q, Pao GM, Huynh AM, Suh H, Tonnu N, Nederlof PM, Gage FH, Verma IM: BRCA1 tumour suppression occurs via heterochromatin-mediated silencing. Nature 2011, 477:179-184.

doi:10.1186/bcr3118

Cite this article as: Elia AEH, Elledge SJ: BRCA1 as tumor suppressor: lord without its RING? Breast Cancer Research 2012, 14:306. 